\title{
Die politische Agenda Deutschlands
}

\author{
Christian Breunig (1) - Tinette Schnatterer
}

Online publiziert: 14. Februar 2020

(C) Der/die Autor(en) 2020

Zusammenfassung Der vorliegende Beitrag stellt eine neue, umfassende Datenbank über die politischen Agenden Deutschlands vor und zeigt ihr Potenzial in der politikwisssenschaftlichen Forschung auf. Dazu fassen wir im ersten Teil den theoretischen Hintergrund - Agendasetzung - kurz zusammen und stellen anschließend die Datenbank vor. Die Datenbank beinhaltet politische Aktivitäten aus allen Etappen des Policyprozesses: die Inputebene (Öffentliche Meinung und Wahlprogramme der Parteien), die Politikprozessebene (Regierungserklärungen, parlamentarische Anfragen und Gesetzesentwürfe) und die Outputebene (Gesetze). Beobachtungen aus jeder Ebene sind thematisch nach dem Schema des Comparative Agendas Project (CAP) codiert und umfassen den Zeitraum von 1977 bis 2013. Schließlich beschreiben wir die gesamte Datenbank kurz und illustrieren am Beispiel der Energiepolitik, wie dadurch politische Prozesse abgebildet und analysiert werden können. Die Datenbank ist zugänglich unter https://gpa.uni-konstanz.de/.

Schlüsselwörter Politische Prozesse - Agendasetzung · Public Policy · Politikfeldanalyse $\cdot$ Deutschland

C. Breunig $(\bowtie)$

Konstanz, Deutschland

E-Mail: christian.breunig@uni-konstanz.de

T. Schnatterer

Pessac Cedex, Frankreich 


\section{German Policy Agenda}

Abstract In this article, we introduce a new and comprehensive database that traces the policy agenda in Germany and highlights its potential for political science research. First, we briefly summarize the theoretical roots-agenda-setting - and then present the database. The database covers activities from all stages of the policy process: at the input level (public opinion and election manifestos of the parties), at the policy process level (government statements, parliamentary questions, and bills), and at the output level (laws). Observations from each level are coded thematically according to the Comparative Policy Agendas scheme and includes the period 1977 to 2013. Finally, we briefly describe the complete database and, using the example of energy policy, illustrate how policy processes can be depicted and analyzed in this way. The database is available at https://gpa.uni-konstanz.de/.

Keywords Political processes $\cdot$ Agenda setting $\cdot$ Public policy $\cdot$ Policy field analysis · Germany

\section{Einleitung}

Der vorliegende Beitrag stellt eine neue, umfassende Datenbank über die politische Agenda Deutschlands vor und skizziert Möglichkeiten, diese Datenbank für die politikwissenschaftliche Forschung zu nutzen. Die Policy-Agendas-Datenbank bietet ein vielfältiges Potenzial für die Beantwortung wichtiger Fragen bezüglich der Entwicklung des politischen Systems Deutschlands und der vergleichenden Politikwissenschaft. Um den Umfang der Daten zu verdeutlichen, zeigen wir auf, wie sich diese Dynamiken in den einzelnen politischen Agenden abbilden und dadurch zu einem besseren Verständnis der Politikgestaltung führen.

Dazu fassen wir in einem ersten Teil den theoretischen Hintergrund - Agendasetzung - kurz zusammen und leiten daraus ihr Potenzial für die Forschung in Public Policy und Politikwissenschaft ab. Anschließend stellen wir die Datenbank vor, die unter https://gpa.uni-konstanz.de/ öffentlich zugänglich gemacht wird. Diese Datenbank enthält alle Etappen des Policyprozesses: die Inputebene, die Politikprozessebene und die Outputebene. Sie umfasst den relativ langen Zeitraum von 1977 bis 2013. Jede Beobachtung wurde thematisch mithilfe des Codierschemas des Comparative Agendas Project (CAP) codiert. Schließlich illustrieren wir am Beispiel der Energiepolitik, welchen Beitrag die vorgestellte Policy-Agendas-Datenbank für die Analyse einzelner Politikfelder spielen kann.

\section{Agendasetting: Das Konzept der Themenaufmerksamkeit}

In parlamentarischen Demokratien koexistieren verschiedene politische Agenden: die Agenda der Regierung, des Parlaments, der Parteien, der Opposition und der öffentlichen Meinung der BürgerInnen. Zusammengenommen formen sie die politische Agenda eines politischen Systems, die ,,alle Probleme [umfasst,] denen die 
Gesellschaft zu einem bestimmten Moment gegenübersteht" und die wiedergibt, „wie die Regierung ihre Aktivität auf ausgewählte Bereiche konzentriert“ (John et al. 2013, S. 1). Agendasetzung ist damit ein Prozess, der zwischen relativ stabilen Elementen politischer Systeme, wie Verfassungen und allgemeinen institutionelle Regelungen, und den volatilen Tagesgeschäften, die durch öffentliche Meinung und politische Debatten bestimmt werden, verankert werden kann.

Agendasetzung wird häufig mit Policystudien in Verbindung gesetzt, die das Aufkommen und die Entwicklung von Themenaufmerksamkeit in einer oder mehreren Agenden analysieren. Agendasetzung sollte jedoch auch als Politics - politischer Konflikt und politisches Handeln - verstanden werden. Unter diesem Blickwickel steht der Einfluss politischer Prozesse und Akteure auf Themenaufmerksamkeit im Zentrum der Analyse. Dieser neue Blickwickel eröffnet die Möglichkeit, den Einfluss institutioneller Faktoren (z. B. zweite Kammer), von Problemen (,Flüchtlingskrise“) sowie von verschiedenen Akteuren (z. B. neuen Parteien) auf Themenaufmerksamkeit zu untersuchen. Ein umfassenderes Verständnis von Agendasetzung bindet Politikinhalte in die breite politikwissenschaftliche Forschung ein und schafft dadurch die Voraussetzungen für eine Vielzahl neuer Forschungsfragen. Um diese Forschungsfragen erfolgreich zu beantworten, fehlte bisher jedoch eine Datenbank, die politische Aktivitäten von der Entstehungsphase bis zum finalen Gesetzesbeschluss abdeckt und alle Politikfelder umschließt. Eine Datenbank, die so umfassend aufgebaut ist, kann den gesamten Agendassetzungsvorgang zu jedem Zeitpunkt der deutschen Politik abbilden. Mithilfe der Datenbank können Politikinhalte dazu genutzt werden, politische Prozesse nachzuzeichnen sowie Konflikte und Handlungsmuster zu identifizieren und $\mathrm{zu}$ analysieren.

\subsection{Agendasetzung in der Policyforschung}

Die Policyforschung hat sich international zu einem der bedeutendsten Bereiche der Politikwissenschaft entwickelt (Castles 2002). Dies gilt auch für Deutschland (Greven 2008, S. 25), wo dieser Trend vor allem Ausdruck in der Entwicklung der Politikfeldanalyse gefunden hat (Janning und Toens 2008; Schneider und Janning 2006). Innerhalb der Politikfeldanalyse, die klassischerweise alle Etappen des Policyzyklus umfasst (Jann und Wegrich 2009), liefert die Untersuchung, wann und wie ein Thema auf die politische Agenda kommt, zentrale Erkenntnisse über Politikinhalte (Downs 1972; Cobb und Elder 1971; Baumgartner und Jones 1993). Ein Beispiel hierfür ist die Studie von Wenzelburger (2015), der Agendasetzung im Bereich der inneren Sicherheit untersucht und aufzeigt, wie sich dieser Politikbereich seit den 1990ern in den meisten OECD-Ländern verändert hat.

Agendasetzungslogiken werden zunehmend als Erklärungsmuster für rapiden Politikwechsel genutzt (Baumgartner et al. 2009). Während das politische System Deutschlands typischerweise als reformunfähig bezeichnet wurde (Rudzio 2005; Schmidt 1987), haben in den letzten Jahren einige sehr weitreichende Politikveränderungen stattgefunden (Rüb 2014). Agendasetzung kann dazu beitragen, solche Veränderungen in Deutschland zu erklären und warum diese eventuell in anderen Ländern ausgeblieben sind. 
Der Beitrag der Agendasetzungsforschung zur Policyforschung beschränkt sich jedoch nicht nur auf den Erkenntnisgewinn in einzelnen Politikfeldern. Eine ihrer Hauptbeiträge liegt vielmehr darin, einen Überblick über die Dynamik innerhalb und das Wechselspiel zwischen allen Politikfeldern zu bieten. Denn wie von Beyme betont, besteht ansonsten die Gefahr, dass ,die Politikwissenschaft sich in die Nischen der Politikfelder [zurückzieht,] ohne zusammenfassenden Ausblick auf das ganze politische System“ (Beyme 1990, S. 21) zu geben. Agendasetzungsforschung erlaubt also nicht nur zu analysieren, wann ein bestimmtes Politikfeld wie auf die Agenda kommt und wer dazu beiträgt, sondern auch die Dynamik der gesamten politischen Agenda zu untersuchen. Damit ermöglicht die Agendasetzungsforschung dem von von Beyme identifizierten Problem zu begegnen, dass die Politikfeldanalyse dazu neigt, ,recht generelle Schlüsse aus immer kleineren Forschungsgegenständen“ zu ziehen (Beyme 1990, S. 21).

Gleichzeitig liefert Agendasetzungsforschung nicht nur Erkenntnisse über die Entwicklung von Themenaufmerksamkeit, sondern ebenso über die Mechanismen, durch die Themen an Bedeutung gewinnen oder verlieren. Beobachtungen, wie Themen durch das politische System ,wandern“, ermöglichen Einflussströme aufzudecken und politische Macht zu lokalisieren. Um eine von Green-Pedersen und Walgrave geprägte medizinische Metapher zu benutzen, ist die Beobachtung von Themenbewegungen in einem politischen System vergleichbar mit dem Injizieren einer Tracerflüssigkeit in einen lebenden Körper, um die Zirkulation der Körperflüssigkeiten zu messen und Schwachstellen zu identifizieren (Green-Pedersen und Walgrave 2014, S. 9).

Ein weiterer Vorteil der Agendasetzungsstudien liegt in der Bereitstellung geeigneter Indikatoren für zentrale politikwissenschaftliche Konzepte. Drei Beispiele verdeutlichen dies: Das erste Beispiel ist die Messung politischer Outputs durch Staatsausgaben (z.B. Cameron 1978; Armingeon 2013). Während Staatsausgaben unbestreitbar ein wichtiger Ausdruck von Regierungsprioritäten sind, wurde in der Literatur jedoch auf mehrere Beschränkungen dieses Indikators hingewiesen (so z. B. die Nichtbeachtung von regulativen Maßnahmen, die Missinterpretation einiger Politikbereiche und Schwierigkeiten bei der Vergleichbarkeit von Zeit und zwischen Ländern). Daten bezüglich der legislativen Aktivität über alle Politikbereiche hinweg enthalten hingegen umfassende Informationen über Prozess, Ziele und Instrumente kollektiver Entscheidungen.

Als zweites Beispiel stellen Regierungserklärungen eine zuverlässige Abbildung von Regierungsprioritäten dar. Regierungserklärungen spiegeln die politischen Intentionen der Regierung wider (Mortensen et al. 2011). Im Gegensatz zu Parteiprogrammen ermöglichen sie die Messung von Regierungsintentionen auch zwischen Wahlen und gehen auf aktuelle Ereignisse, Entwicklungen und die Erwartungen der BürgerInnen ein (Breunig et al. 2019).

Schließlich eröffnen Agendasetzunsgdaten neue Einsichten in den Parteienwettbewerb und in die von den Parteien gewählten Strategien (John et al. 2013). Unter anderem ermöglichen Agendasetzungsdaten den politischen Wettbewerb nicht nur durch Parteiprogramme, sondern auch durch politische Reden und legislative Instrumente zu messen (Proksch und Slapin 2015). 
Insgesamt wird durch diese Beispiele klar, dass die Agendasetzungsforschung Fragen über Institutionen und Akteure aus einer Prozessperspektive stellt. So werden zum Beispiel Parteien nicht als formale Akteure behandelt, sondern diese werden ,auf frischer Tat ertappt“ (Green-Pedersen und Walgrave 2014, S. 11), indem beobachtet wird, wie sie die Prioritätensetzung politischer Themen beeinflussen.

\subsection{Agendasetzung aus dem Politics-Blickwinkel}

Formelle und informelle Regeln schaffen Foren für politischen Wettbewerb. Deshalb besitzen Institutionen eine wichtige Rolle in der Agendasetzungsforschung. Sie generieren einerseits Räume, die danach verlangen mit Aufmerksamkeit ausgefüllt zu werden, und geben andererseits die Grenzen vor, wieviel Aufmerksamkeit verschiedenen Themen insgesamt zuteilwerden kann (Green-Pedersen und Walgrave 2014). Eine systematische Analyse der Art und Weise, wie politische Institutionen und Akteure ein Thema aufgreifen und bearbeiten, liefert daher theoretische Erkenntnisse und wichtige empirische Befunde über das politische System Deutschlands.

Der Zugang zur Abbildung politischer Prozesse und kollektiven Handels wird durch die politischen Themen geschaffen. Durch den Rückgriff auf die ,gemeinsame Währung Themenaufmerksamkeit" (Green-Pedersen und Walgrave 2014, S. 10) können verschiedene Themen, verschiedene Agenden und politische Systeme verglichen werden. Die Möglichkeit, die Interaktionen zwischen so verschiedenen Aktivitäten wie der Gesetzgebungstätigkeit, dem Stellen parlamentarischer Anfragen, der Umsetzung von Parteiprogrammen oder des Einflusses von Meinungsumfragen zu untersuchen, versetzt Studien der Agendasetzung in die Lage, nahezu das ganze politische System zu erfassen. Dies gilt insbesondere für die vergleichende Forschung. In beiden Fällen bietet das Abbilden von politischen Themen, sei es die Rentenpolitik oder der Atomausstieg, das Potenzial, das Zusammenspiel von politischen Interessen und Institutionen noch besser zu verstehen.

Dabei ist nicht die Wichtigkeit eines bestimmten politischen Themas der zentrale Untersuchungsgegenstand, stattdessen wird erforscht, ob und wie ein politisches System es schafft, gesellschaftliche Probleme zu bearbeiten und zu lösen. Da die Fähigkeit gesellschaftliche Probleme zu lösen stark durch Interaktionen zwischen Akteuren und Institutionen bestimmt wird (Scharpf 1997), tragen Untersuchungen zur Agendasetzung dazu bei, politische Handlungsmuster zu identifizieren und zu erklären. Genau durch diese Möglichkeit schafft es die Agendasetzungsforschung, zentrale Fragen der Politikwissenschaft bezüglich des Parteienwettbewerbs (Klüver und Spoon 2016; Breunig et al. 2019), der demokratischen Responsivität (Wlezien 2017) und der Repräsentation (Bailer et al. 2018) aufzugreifen. Unser Ziel ist es, eine umfassende Datenbank zur Verfügung zu stellen, die diese Prozessperspektive ermöglicht und fördert, um dadurch zentrale Fragen zur deutschen Politik zu beantworten. 


\section{Politische Agenden in Deutschland - die Datenbank}

Individuelle politische Agenden, im Besonderen in einzelnen Politikfeldern, sind typischer Forschungsgegenstand der Policyforschung. So zum Beispiel in der Moralpolitik (Heichel et al. 2015), in der Gesundheitspolitik (Landwehr und Böhm 2016), im Hinblick auf Verfassungsreformen (Behnke 2009), während Wahlkämpfen (Brettschneider 2004) oder in Bezug auf politische Parteien in Deutschland (Bräuninger und Debus 2011).

Zumeist konzentrieren sich diese Studien auf eine begrenzte Anzahl von Agenden und Themen. Dies kann durch das Design begründet werden, aber auch der Tatsache geschuldet sein, dass noch keine leicht zugängliche und umfassende Datenbank besteht, die alle politischen Agenden über einen langen Zeitraum erfasst. Die hier vorgestellte Datenbank schließt diese Lücke. Alle politischen Aktivitäten innerhalb einer Agenda werden erfasst und jede Beobachtung wird einem thematischen Code zugeordnet. Damit liefert das deutsche Agendaprojekt die Möglichkeit von Vergleichen sowohl über die Zeit als auch zwischen den einzelnen Agenden. Jede Beobachtung in der Datenbank ist eindeutig durch eine Datumsangabe und einen Dokumentindikator, wie Umfrage- oder Gesetzesnummer, identifiziert und kann damit auch mit weiteren Kontextdaten zusammengeführt werden. In diesem Abschnitt stellen wir das Klassifikationsschema, die zugrundeliegenden Daten und das Codierungsverfahren vor.

Auf internationaler Ebene bringt das Comparative Agendas Project (CAP) Daten zu Regierungsaktivitäten, Medienberichterstattung, öffentlicher Meinung, Parteien etc. in über 20 Ländern zusammen (Baumgartner et al. 2019). Zumeist sind diese länderspezifischen Daten auch über einen längeren Zeitraum zusammengefasst. Aufgrund der open-data Philosophie und dem großen Wert, der auf Vergleichbarkeit durch gemeinsame Codierentscheidungen gelegt wird, bieten die CAP-Datensätze eine beispielslose Grundlage für internationale Vergleiche. ${ }^{1}$

\subsection{Klassifikationsschema}

Unser Klassifikationsschema folgt dem Comparative Agendas Project ${ }^{2}$, welches politische Aktivitäten thematisch in 21 Hauptkategorien und 232 Unterkategorien einteilt. Die 21 Kategorien bestehen aus folgenden Themen: Volkswirtschaft, Bürgerrechte, Gesundheitswesen, Landwirtschaft, Arbeitswesen, Bildung, Umwelt, Energie, Transport, Innere Sicherheit, Sozialpolitik, Wohnungswesen, Bank-/ Finanzwesen, Verteidigung, Wissenschaft/Technik, Außenhandel, Internationale Beziehungen, Öffentliche Verwaltung, Öffentliches Land, Länder und Kommunen und Wiedervereinigung. Diese Kategorien schließen sich gegenseitig aus (d.h. jede Beobachtung kann nur einem einzigen Code zugeordnet werden) und sind insgesamt vollständig (d.h. jede Beobachtung kann tatsächlich einem bestimmten Thema zugeordnet werden). Es wurden bewusste Anstrengungen unternommen, um die

\footnotetext{
${ }^{1}$ Siehe https://www.comparativeagendas.net für eine Beschreibung des Projekts.

2 https://www.comparativeagendas.net/datasets_codebooks für den direkten Zugang zum Codierschema des CAP-Projekts.
} 
zeitliche Konsistenz dieser Kategorien zu gewährleisten, d.h. das Codierschema ist zeitkompatibel.

Diese drei Eigenschaften - Exklusivität, Vollständigkeit und Zeitkompatibiliät - bieten einen entscheidenden Vorteil gegenüber den typischen, von Institutionen, Archiven etc. vergebenen Schlagworten, die häufig über die Zeit angepasst oder verfeinert werden. Der Ursprung des Codierschemas liegt im amerikanische Codebuch, welches in einem iterativen Prozess erstellt wurde, um alle Politikfelder abzubilden (Baumgartner und Jones 2002, S. 35). Jede der 21 Kategorien enthält mehrere Unterkategorien sowie eine allgemeine Unterkategorie, die Fälle enthält, auf die mehrere Unterkategorien zutreffen. Die feingliedrige Einteilung der Unterkategorien erlaubt Nutzern, passend zu der jeweiligen Forschungsfrage, bestimmte Themen zusammenzufassen. So lassen sich zum Beispiel Unterkategorien aus den Bereichen Umwelt und Energie oder Arbeit und Sozialpolitik zusammenstellen.

Eine Besonderheit des deutschen Codebuchs ist eine Kategorie für die deutsche Wiedervereinigung. Dokumente wurden der Kategorie Wiedervereinigung zugeordnet, wenn diese explizit erwähnt wurde oder der Inhalt eindeutig auf die Folgen der Wiedervereinigung Bezug nahm. Wir haben uns für dieses Vorgehen entschieden, um sowohl dem einzigartigen Charakter dieses historischen Prozesses als auch den politischen Herausforderungen des neuen Deutschlands gerecht zu werden.

\subsection{Datenreihen}

Im Folgenden unterteilen wir den Politikzyklus in Inputs (Umfragen zur öffentlichen Meinung und Wahlprogramme der Parteien), politische Prozesse (Regierungserklärungen, parlamentarische Anfragen und Gesetzesentwürfe) und Outputs (Gesetze) und bilden diesen Zyklus mit den in Klammern angegebenen Dokumenten ab. Der Datensatz umfasst den Zeitraum von 1986 bis 2013 für die Umfragedaten, 1976 bis 2013 für Parteiprogramme und die 8.-17. Wahlperiode des Bundestags für alle anderen Dokumente (Dezember 1976 bis Oktober 2013). Außer den Umfragedaten und den Wahlprogrammen basieren alle Daten auf Informationen des Dokumentationsund Informationssystems für Parlamentarische Vorgänge (DIP).

Die öffentliche politische Agenda messen wir mithilfe von Daten zur öffentlichen Meinung und konzentrieren uns dabei auf die Antworten auf die Frage nach dem gegenwärtig wichtigsten Problem in Deutschland (im Folgenden beziehen wir uns auf diese Frage mit „MIP“, von „,most important problem“ im Englischen). Diesen MIPDatensatz haben wir aus den Datensätzen der jährlichen Zusammenfassung des Politbarometers erstellt. Das GESIS-Leibniz Institut für Sozialwissenschaften stellt die Daten über das ZACAT-Portal öffentlich zur Verfügung. Pro Jahr werden zwischen 11.000 und 25.000 BürgerInnen der alten Bundesländer und seit 1991 durchschnittlich 11.000 in den neuen Bundesländern in meist monatlichen Intervallen von der „Forschungsgruppe Wahlen e. V.“ befragt. Die Antworten auf die offene Frage werden durch das Politbarometerteam in generelle Kategorien zusammengefasst. Anstatt den von GESIS zur Verfügung gestellten kumulativen Datensatz zu nutzen, haben wir alle einzelnen monatlichen Umfragewellen neu zusammengestellt, um eine differenziertere Codierung zu gewährleisten. Die von uns verwendete Variable ist seit 1980 in den Umfragen enthalten, seit 1986 in regelmäßigen Abständen. 
Die Inputserie enthält außerdem die Wahlprogramme für acht Bundestagswahlen zwischen 1976 und 2013. Die Wahlprogramme aller fünf in diesem Zeitraum im Bundestag vertretenen Parteien (CDU/CSU, FDP, Grüne, Linke/PDS und SPD) wurden unter der Leitung von Christoffer Green-Pedersen (Aarhus University) und Isabelle Guinaudeau (Sciences Po Bordeaux) auf Satzebene codiert (Guinaudeau 2015). Typisch für Wahlprogramme ist, dass sie über die Zeit umfangreicher werden.

Als Indikator der Regierungsagenda nutzen wir Regierungserklärungen. Für unsere Datenbank haben wir für jedes Jahr die wichtigste Regierungserklärung des Bundeskanzlers und der Bundeskanzlerin identifiziert. Regierungserklärungen, die lang sind und mehrere Themenbereiche abdecken, wurden gegenüber Reden, die kurz und monothematisch sind, bevorzugt. Durch dieses Vorgehen ist unser Datensatz weniger Ereignis-geleitet, als wenn wir alle Erklärungen des Bundeskanzlers berücksichtigt hätten und ermöglicht dennoch der Verschiebung und Entwicklung von Prioritäten zwischen Wahlen Rechnung zu tragen. Dies ist umso wichtiger, als die bisherige Literatur zu Regierungserklärungen des Bundeskanzlers sich auf die Antrittsreden zu Beginn einer Legislaturperiode konzentriert (s. z. B. Von Beyme 1979; Stüwe 2002). Jeder Satz wurde in Quasisätze unterteilt und diese dann thematischen Codes zugeordnet. Laut der Manifesto-Gruppe (Budge et al. 2001, S. 215-219) sind Quasisätze einzelne Ausdrücke politischer Ideen oder Themen. Beinhaltet ein Satz also zwei oder mehr Aussagen werden diese in einzelne thematische Einheiten zerlegt.

Die politische Agenda der Abgeordneten wird in unserer Datenbank durch Große und kleine parlamentarische Anfragen erfasst. Diese sind ein parlamentarisches Instrument, das typischerweise von Parteien und Abgeordneten der Opposition genutzt wird. Formal kann jede Fraktion oder fünf Prozent aller Abgeordneten eine parlamentarische Anfrage stellen (Regel 75-76, Geschäftsordnung des Deutschen Bundestages). Empirisch kann festgestellt werden, dass dieses Instrument vor allem von den Oppositionsparteien und darunter vor allem von den Grünen und den Linken/PDS genutzt wird: So kamen zum Beispiel $62 \%$ der kleinen Anfragen in der 14. Wahlperiode aus den Reihen der Abgeordneten der Linken. Als Codiergrundlage dienen der Titel, die Schlagwörter und die vom DIP zur Verfügung gestellten Zusammenfassung der Anfrage. Wenn es nötig war, wurde zusätzlich der komplette Inhalt der Anfrage zur Hilfe gezogen.

Ein Datensatz, der alle eingebrachten Gesetzesentwürfe enthält, erlaubt in verabschiedete und nicht verabschiedete Gesetze zu unterscheiden. Die Agenda des gesamten Bundestags findet Ausdruck in den eingebrachten Gesetzesentwürfen. Diese können von der Regierung, dem Bundesrat oder fünf Prozent aller Bundestagsabgeordneten eingebracht werden (Art. 76, GG). Die verabschiedeten Gesetze hingegen werden typischerweise als ein Indikator des politischen Outputs angesehen. Somit ist es möglich, zwischen verabschiedeten und nicht verabschiedeten Gesetzesentwürfen zu unterscheiden: Für die Codierung der Gesetzesentwürfe und der verabschiedeten Gesetze wurden der Titel, die Schlagwörter und die Zusammenfassung sowie der gesamte Text verwendet. 


\subsection{Codierverfahren}

Das Ziel der Codierung ist eine eindeutige, thematische Zuordnung der jeweiligen Beobachtung (z. B. Satz oder Dokument) nach dem Politikinstrument. Es wurde also nicht die Zielpopulation codiert und auch nicht das intendierte Ziel einer Maßnahme, sondern das politische Instrument. So werden zum Bespiel Fragen bezüglich der Renten von medizinischem Personal in „Renten“ und nicht in „,medizinisches Personal" codiert.

Das Codierverfahren lief folgendermaßen ab: Alle Dokumente wurden von mindestens zwei gut ausgebildeten Codierern zugeordnet. Diese Codierentscheidungen wurden im zweiten Schritt von einer dritten Person durchgesehen. Im dritten Schritt wurden alle Beobachtungen gemeinsam evaluiert. Im Falle von Uneinigkeiten wurden diese gemeinsam diskutiert und anschließend exklusiv einer Kategorie zugeordnet.

Aufgrund des hohen Datenvolumens und der Tendenz einiger Abgeordneter und Parteien ähnliche Anfragen zu stellen, wurden die parlamentarischen Anfragen der 11.-15. Wahlperiode mithilfe von RTextTools halbautomatisch codiert (Jurka et al. 2012; Grimmer und Steward 2013). Die halbautomatische Codierung wurde mit Zuhilfenahme elektronisch verfügbarer Textinhalte (Titel, Zusammenfassung etc.) codiert und dabei nicht der gesamte Orginaltext konsultiert. Die verwendeten Algorithmen wurden mit einer manuell erstellten Trainingsdatei trainiert. ${ }^{3}$ Anschließend wurden diejenigen Anfragen bei denen mindestens zwei Algorithmen übereinstimmten (meist SVM und Maxent) in der üblichen Codierprozedur von zwei gut ausgebildeten Codierern verifiziert. Im Falle von Uneinigkeiten zwischen den drei (Codierern und Algorithmen) wurden diese wieder gemeinsam diskutiert, um eine Anfrage nur einer bestimmten Kategorie zuzuordnen. Abschließend wurden alle Fälle, in denen die Algorithmen nach vier Codierdurchläufen nicht zu einer Einigung kamen, manuell codiert. Die halbautomatische Codierung erwies sich am sichersten für Anfragen im Bereich Umwelt und am schwierigsten für Anfragen zum Thema Bank-/Finanzwesen.

Die deutsche Policy-Agendas-Datenbank beinhaltet thematische Informationen aus den sechs in Abschn. 2.2 dargestellten Datenreihen. Sie bietet dadurch einen umfassenden Überblick über alle in Deutschland diskutierten Policythemen für die Jahre 1977-2013. Während des Beobachtungszeitraums wurden insgesamt 769.681 Einheiten klassifiziert. Die Datenbank erlaubt daher eine weitreichende Projektion des politischen System Deutschlands.

Tab. 1 beinhaltet eine Übersicht über die insgesamt sechs Datenserien: Im Bereich Input sind 655.182 Antworten auf die Frage nach dem wichtigsten Problem codiert sowie 68.194 Sätze in Wahlprogrammen. Die Inter-Coder-Reliabilität beträgt 94,1\% für MIPs und 97,9\% für Wahlprogramme. Der Bereich des Policyprozesses umfasst 17.265 Quasisätze aus Regierungserklärungen, 17.081 parlamentarische An-

\footnotetext{
3 Die Trainingsdatei wurde in einem iterativen Prozess erstellt. In einem ersten Schritt wurden handcodierte Beobachtungen aus der vorhergehenden Legislaturperiode sowie 300 zufällig ausgewählte und handcodierte Beobachtungen aus der zu codierenden Legislaturperiode als Trainingsbasis verwendet. Für jeden weiteren Durchlauf wurden dann die bereits codierten und verifizierten Codes hinzugefügt.
} 
Tab. 1 Erfasste politische Agenda in Deutschland

\begin{tabular}{|c|c|c|c|c|c|}
\hline & Indikator & Akteure & Analyseeinheit & $\begin{array}{l}\text { Anzahl } \\
\text { Beobach- } \\
\text { tungen }\end{array}$ & $\begin{array}{l}\text { Inter-Coder- } \\
\text { Reliabilität } \\
\text { WP 8-17 }\end{array}$ \\
\hline \multirow[t]{2}{*}{ Input } & $\begin{array}{l}\text { Wichtigstes } \\
\text { Problem } \\
\text { (MIP) }\end{array}$ & $\begin{array}{l}\text { Teilnehmer repräsen- } \\
\text { tativer Umfragen }\end{array}$ & $\begin{array}{l}\text { Antwort } \\
\text { auf offene } \\
\text { Frage }\end{array}$ & 655.182 & 94,1 \\
\hline & Parteiprogramme & Politische Parteien & Satz & 68.194 & 88,3 \\
\hline \multirow[t]{3}{*}{$\begin{array}{l}\text { Policy- } \\
\text { prozess }\end{array}$} & $\begin{array}{l}\text { Wichtigste Re- } \\
\text { gierungserklä- } \\
\text { rung des Jahres }\end{array}$ & BundeskanzlerIn & Quasisatz & 17.265 & 85,3 \\
\hline & $\begin{array}{l}\text { Große und kleine } \\
\text { Anfragen }\end{array}$ & $\begin{array}{l}\text { Jede Fraktion oder } \\
5 \% \text { aller Abgeordne- } \\
\text { ten }\end{array}$ & Fragetext & 17.081 & 80,7 \\
\hline & Gesetzesentwürfe & $\begin{array}{l}\text { Die Bundesregierung, } \\
\text { der Bundesrat, oder } \\
5 \% \text { aller Abgeordne- } \\
\text { ten }\end{array}$ & $\begin{array}{l}\text { Text des } \\
\text { Gesetzes- } \\
\text { entwurfs }\end{array}$ & 7666 & 83,8 \\
\hline Output & $\begin{array}{l}\text { Verabschiedete } \\
\text { Gesetze }\end{array}$ & $\begin{array}{l}\text { Parlament verabschie- } \\
\text { det Gesetze }\end{array}$ & Gesetzestext & 42.493 & 82,3 \\
\hline
\end{tabular}

fragen und 7666 Gesetzesentwürfe, mit einer jeweiligen Inter-Coder-Reliabilität von $85,3 \%, 80,7 \%$, und 83,8\%. Im Bereich der Outputs haben wir 4293 Gesetze mit einer Inter-Coder-Reliabilität von $82,3 \%$ codiert.

Die vorgestellte Datenbank ist in deutscher Sprache unter https://gpa.unikonstanz.de/ zugänglich. Außerdem wird sie auf GESIS archiviert. In Zukunft soll die Datenbank nach Abschluss einer jeweiligen Legislaturperiode und der öffentlichen Bereitstellung aller Daten durch den Bundestag aktualisiert werden.

\section{Politische Agenden in der BRD}

Im Folgenden geben wir einen kurzen Gesamtüberblick über die Datenbank und beschreiben an einem Beispiel aus dem Politikfeld Energiepolitik, welche Informationen unsere Datenbank beinhaltet und wie man diese nutzen kann. Ziel dieser Skizze ist es nicht, neue Erkenntnisse zu gewinnen, sondern vielmehr das Potenzial unserer feingliedrigen Daten darzustellen. Insbesondere das Beispiel der Energiewende stützt sich auf bestehendes Wissen aus Einzelfallstudien, welche unsere Daten validieren.

\subsection{Die Vogelperspektive}

Eine grafische Darstellung bietet eine erste Vorstellung des Aufbaus der vorgestellten Datenbank. Abb. 1 bildet die inhaltliche Zusammensetzung der verschiedenen politischen Agenden über die Zeit ab. Die Wiedergabe der Themenaufmerksamkeit in Prozent trägt dabei der Tatsache Rechnung, dass die potenzielle Aufmerksamkeit einer Agenda begrenzt ist. Gleichzeitig vereinfacht diese Darstellung den Vergleich 


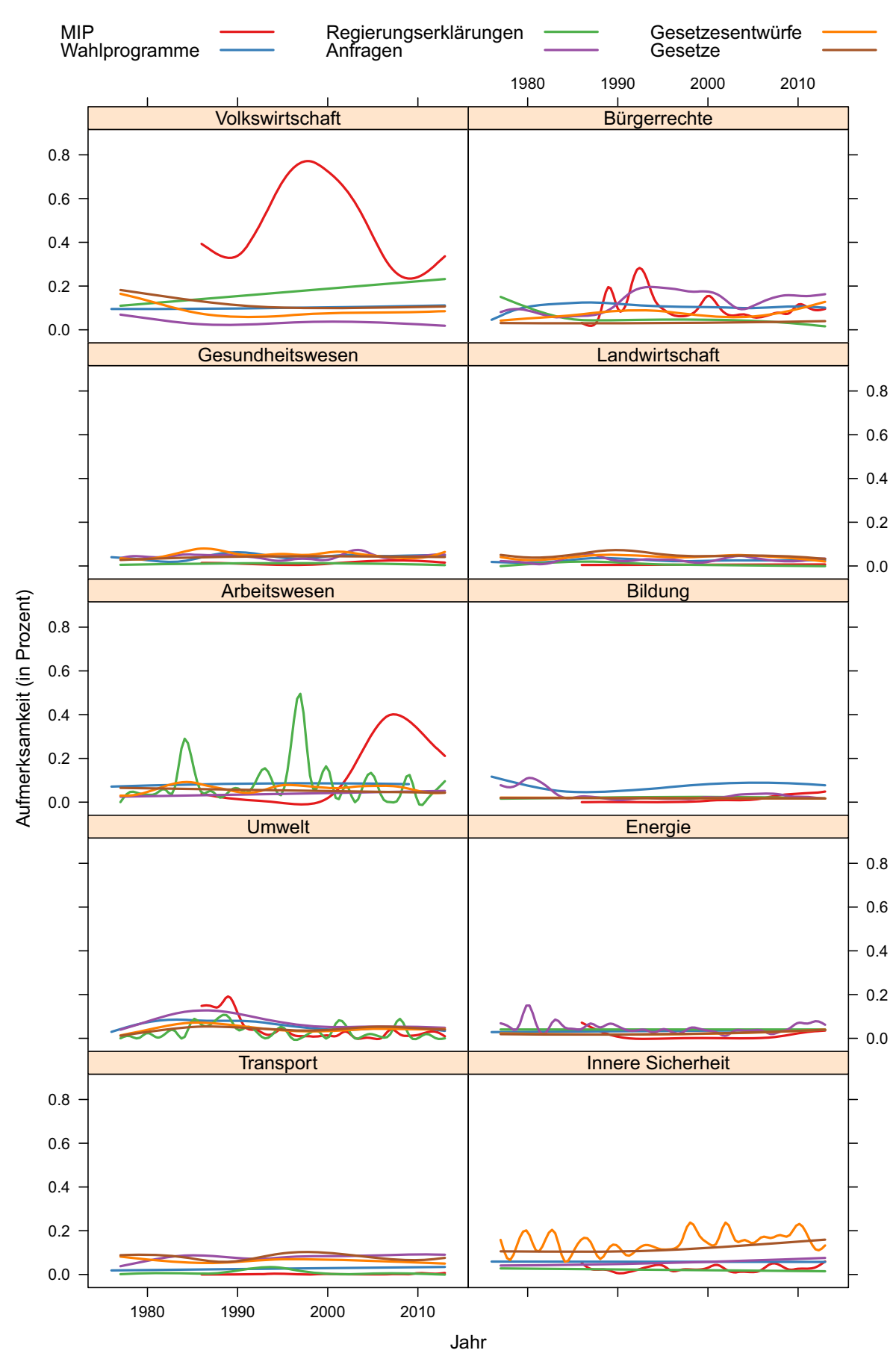

Abb. 1 Themenaufmerksamkeit über die Zeit und Agenden 


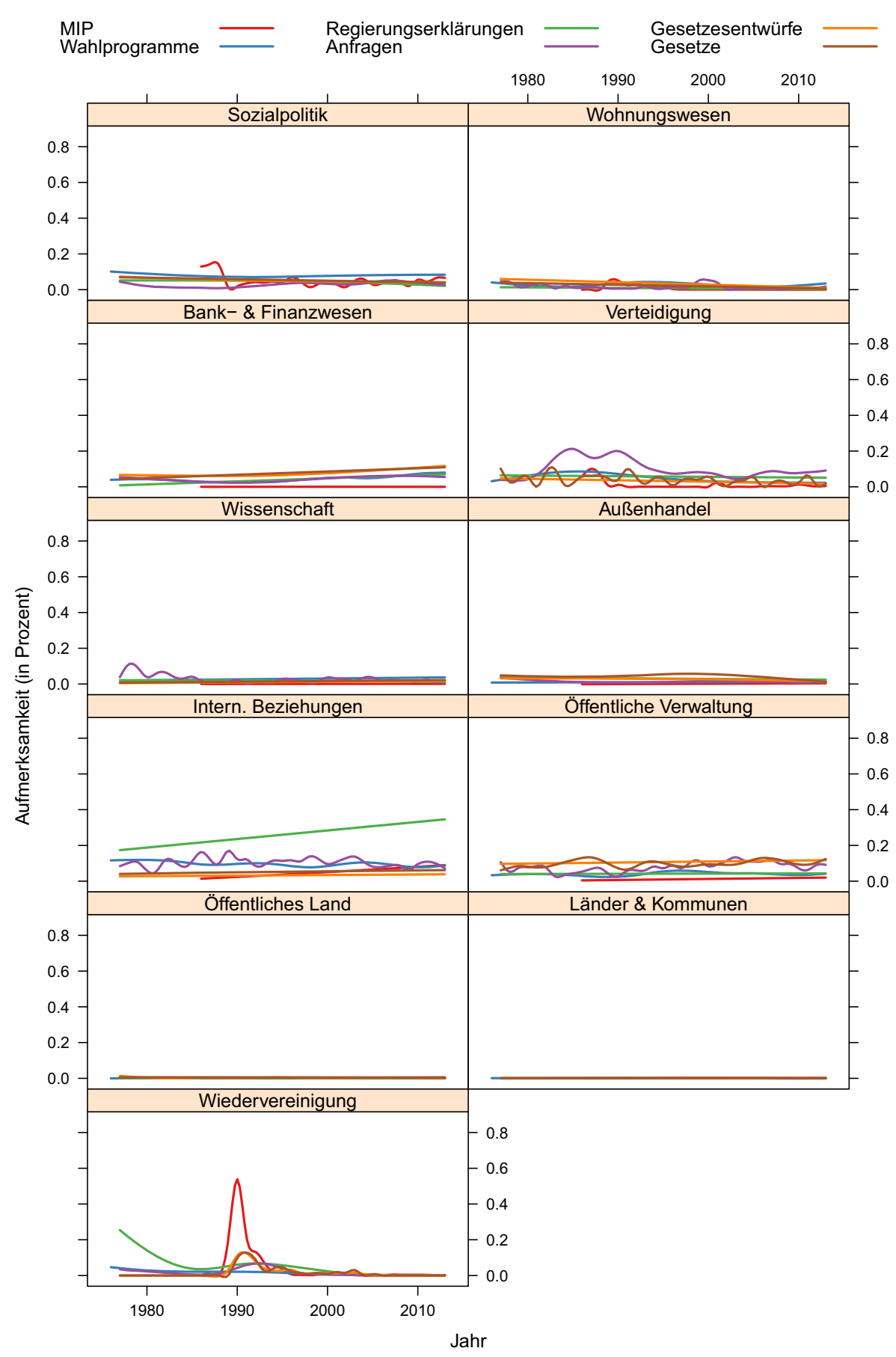

Abb. 1 (Fortsetzung) 
zwischen verschieden großen Agenden. Diese einfache Darstellung verdeckt natürlich viele wichtige Prozesse innerhalb und zwischen Agenden (so verschwinden etwa Veränderungen in der Beobachtungsmenge über die Zeit oder Unterschiede zwischen Parteien in der Nutzung diverser Agendasetzungsinstrumente) $)^{4}$, ist aber als erste Annäherung an die Daten hilfreich.

Eine erste visuelle Interpretation dieser Abbildungen lässt Unterschiede sowohl zwischen verschiedenen Agenden als auch zwischen verschiedenen Themenbereichen erkennen. Im Vergleich zu Umfragen und Regierungserklärungen weist die Summe aller Parteiprogramme nur schwache Veränderungen der Themenaufmerksamkeit über die Zeit auf. Gleichzeitig erreicht zu keinem Zeitpunkt ein einzelnes Thema mehr als $15 \%$ der Gesamtaufmerksamkeit. Die höchste Konzentration ist in den Programmen zur Bundestagswahl 2005 zugunsten des Themas volkswirtschaftliche Steuerung zu verzeichnen. Gesetze und Gesetzesentwürfe weisen ebenfalls eine relative Stabilität auf.

Für parlamentarische Anfragen, eines der Hauptinstrumente der parlamentarischen Opposition, lässt sich in den letzten Jahrzehnten eine Schwerpunktverschiebung gegenüber den 80er-Jahren erkennen. Mit dem Einzug der Grünen in den Bundestag nahmen die Themen Umwelt und Verteidigung eine wichtige Rolle in den Anfragen der 80er-Jahre ein. Dahingegen kam es mit dem Einzug der PDS/ Linken in den Bundestag zu einer Zunahme parlamentarischer Anfragen im Bereich der Bürgerrechte.

Einige der spektakulärsten Wandel der Themenaufmerksamkeit lassen sich für die Antworten auf die ,most important problem“-Frage feststellen. Generell ist zu beobachten, dass diese sehr von Themen im Bereich Volkswirtschaft und Arbeitsmarktpolitik geprägt sind. Immer wieder gewinnen jedoch einzelne andere Themen an Bedeutung, wie zum Beispiel Bürgerrechte Ende der 80er- und Anfang der 90erJahre oder, als Reaktion auf die internationale Banken- und Finanzkrise im Jahr 2009, internationale Beziehungen. Auch die deutsche Wiedervereinigung, die erwartungsgemäß zu einer Zunahme dieses Themas in allen Agenden führte, hat die deutlichsten Auswirkungen auf die Bürgerantworten. So führten $199054 \%$ aller Befragten dieses Thema als gegenwärtig wichtigstes Problem Deutschlands an. Regierungserklärungen unterliegen ebenfalls häufigem Wandel. Auf den ersten Blick scheinen deutsche BundeskanzlerInnen einen Schwerpunkt auf Internationale Beziehungen zu legen. Andere Themen wie Volkswirtschaft, Verteidigung oder Energie spielen jedoch punktuell ebenfalls eine wichtige Rolle.

\subsection{Fokus auf ein Politikfeld: Der Atomausstieg und das Aufkommen alternativer Energien in den politischen Agenden der BRD}

Die Datenbank trägt somit dazu bei, einen Überblick über die Dynamik und das Wechselspiel aller Politikfelder zu bieten und bereichert damit die Forschung zum politischen Systems Deutschlands. Gleichzeitig bietet sie auch vielfältige Möglichkeiten, Studien in einzelnen Politikfeldern zu entwickeln. Durch die feinmaschigere Codierung kann man beispielsweise analysieren, wie sich die Aufmerksamkeit in-

\footnotetext{
4 Wir danken einem anonymen Gutachten für diesen Hinweis.
} 
nerhalb eines Politikfelds zwischen Agenden unterscheidet und wie sich diese über die Zeit verändern.

Ein aktuelles Beispiel hierfür ist die Energiepolitik. Der deutsche Atomausstieg und der plötzliche Positionswechsel der Merkel-Regierung hat international und auch in der wissenschaftlichen Literatur viel Beachtung erhalten. Erklärungen, die diesen einfach auf das Unglück in Fukushima zurückführen (Davies 2011), greifen offensichtlich zu kurz, da sie weder erklären, warum ein ähnlicher Politikwechsel in anderen Ländern ausgeblieben ist, noch warum dieser nicht bereits nach der Katastrophe in Tschernobyl eintrat. Andere Studien verweisen auf politische Motivationsgründe wie die Wahlen in drei Bundesländern oder den politischen Druck durch die Bewegung gegen das Großprojekt Stuttgart21 (Jahn und Koralczak 2012; Wittneben 2012). Diese Studien, die sich in Ansatz und Methode unterscheiden, haben häufig gemein, dass sie sich auf einen relativ kurzen Zeitraum konzentrieren. Eine erste visuelle Interpretation der energiepolitischen Themen (siehe Abb. 2) in den einzelnen politischen Agenden in den letzten Jahrzehnten deckt längerfristige Trends auf und ermöglicht Unterschiede sowie Gemeinsamkeiten zwischen 1986 (Tschernobyl) und 2011 (Fukushima) herauszuarbeiten.

Beide Reaktorunglücke haben gemein, dass sie das Thema Atomenergie und im Besonderen die Sicherheit von Atomkraftwerken verstärkt auf die politische Agenda gebracht haben. Dies verdeutlicht ein Blick auf die verschiedenen Agenden von der Inputebene zur Outputebene. In den Antworten der BürgerInnen auf die Frage nach dem gegenwärtig wichtigsten Problem in Deutschland (MIP), die allgemein sehr von wirtschaftlichen und arbeitsmarktpolitischen Themen geprägt sind, spielen energiepolitische Themen generell kaum eine Rolle. Umso aussagekräftiger ist es, dass im Juni $198623 \%$ der Befragten Atomenergie als wichtigstes Problem in Deutschland nannten (im April und Mai 1986 fanden keine Befragungen statt). Im März 2011, dem Monat der Nuklearkatastrophe in Fukushima, waren es $37 \%$ der Befragten. Beide Unfälle beschäftigten also die öffentliche Meinung. Die Tatsache, dass Deutschland direkter von den Folgen der Katastrophe in Tschernobyl betroffen war, drückt sich vor allem darin aus, dass die Antworten auf die offene Frage in der 1986 Umfrage konkreter waren (,,verstrahlte Molke“). In beiden Fällen wurde die Beunruhigung der Bürger in den Regierungserklärungen sowohl von Bundeskanzler Helmut Kohl (31\% aller Quasisätze) als auch von Bundeskanzlerin Angela Merkel (11\% aller Quasisätze) aufgegriffen.

Zwischen beiden Unglücken hatte sich die energiepolitische Debatte in Deutschland jedoch grundlegend gewandelt. Ein Blick auf den Anteil der Unterkategorien im Bereich Energie zeigt, dass ein neues Themenfeld an Bedeutung gewonnen hat: Die Aufmerksamkeit für alternative Energien hat in allen Agenden im Vergleich zu den 1980ern deutlich zugenommen.

Dieser Trend beginnt vor der Katastrophe in Fukushima und hält im Wesentlichen bis zum Ende der Datenreihe im Jahre 2013 an. In den parlamentarischen Anfragen spielen erneuerbare Energien sogar schon früher eine Rolle. Die relativ hohe Zahl der Anfragen zu erneuerbaren Energien in den 1980ern erklärt sich durch die verstärkte Aktivität der Grünen in diesem Bereich. Anfragen zu alternativen Energien nehmen aber nach Tschernobyl leicht zugunsten von Fragen bezüglich der Atomindustrie ab. Was die in den Bundestag eingebrachten Gesetzesentwürfe betrifft, ist die 


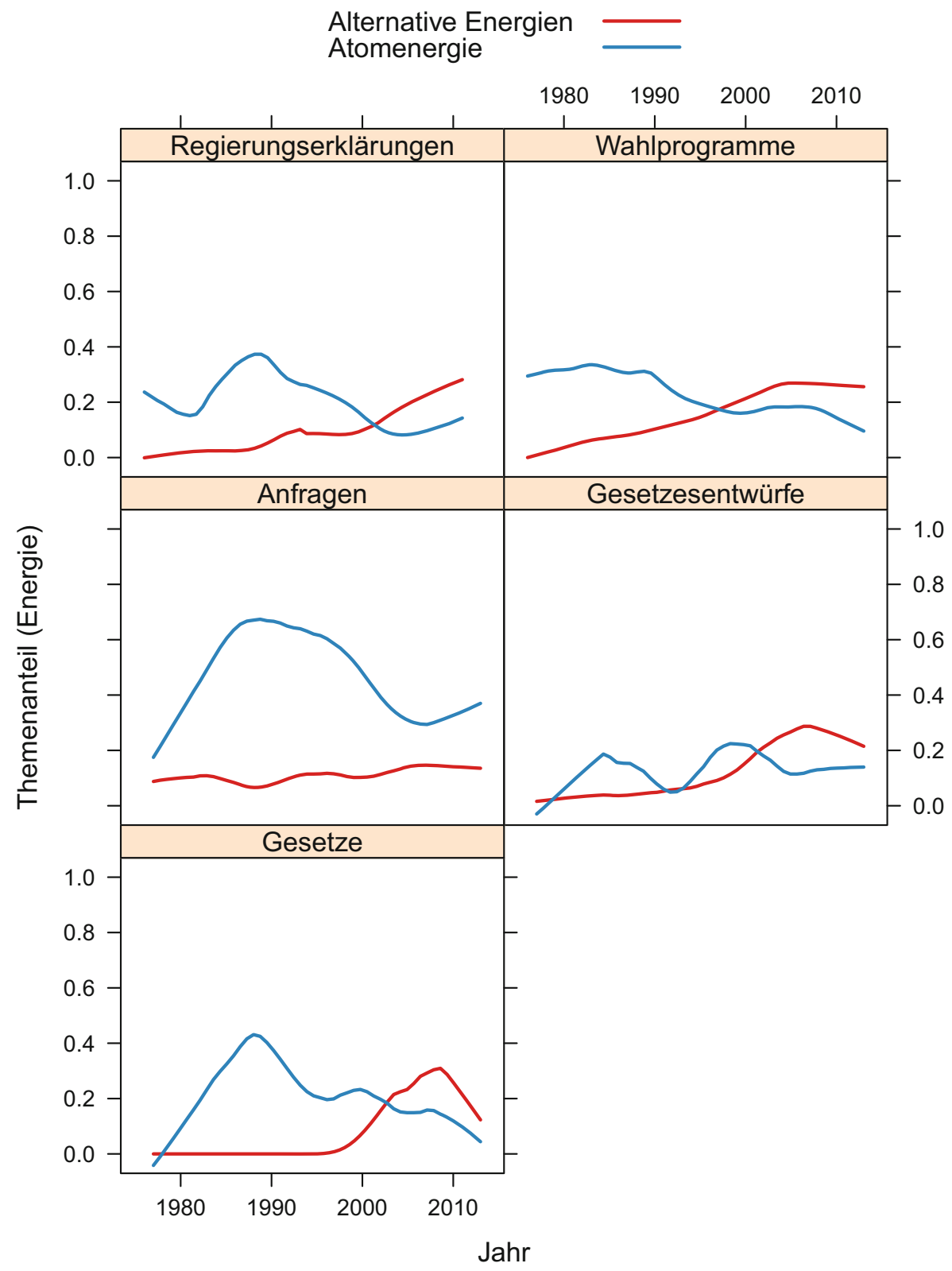

Abb. 2 Anteil der Dokumente zu alternativen Energien bzw. Atomenergie von allen energiepolitischen Themen in Prozent (geglättete Werte) 
Beobachtung eindeutig: Vor dem Stromeinspeisungsgesetz 1990 wurden nur zwei Gesetzesentwürfe zum Thema erneuerbare Energien eingebracht.

Am deutlichsten spiegelt sich das Aufkommen dieses Themas jedoch in den Parteiprogrammen wider. 1987 brachte Tschernobyl zuerst das Thema Atomenergie auf die Agenda der großen Volksparteien: Die CDU hatte dieses Thema in den Bundestagswahlprogrammen davor gar nicht erwähnt, die SPD vertrat zum ersten Mal die Position eines Ausstiegs in zehn Jahren, nachdem sie der Atomkraft bei den vorangegangenen Wahlen positiv gegenübergestanden war. Diese Konfliktlinien bleiben bis 2011 bestehen (SPD, Grüne/Linke für den Atomausstieg, CDU/CSU und FDP dagegen). Auch hier verliert das Thema Atomenergie aber an Bedeutung zugunsten des Themas erneuerbare Energien.

In der Legislaturperiode 1998-2005 wird aus dem Thema Atomausstieg die Energiewende: Die Rot-Grüne Bundesregierung definierte erneuerbare Energien nicht mehr als Ergänzung, sondern als Alternative zu herkömmlichen Energieformen. In der Folge nehmen 2009 alle Parteien in ihren Programmen positiv Bezug auf das Erneuerbare-Energien-Gesetz: Im gleichen Zeitraum gewinnen erneuerbare Energien in den Regierungserklärungen und Gesetzesinitiativen an Bedeutung.

Die kurze Datenauslese macht deutlich: Der politische Wettbewerb unterscheidet sich 2011 immens von dem in 1986. In beiden Fällen ruft ein Reaktorunglück als externer Schock Sorgen in der Bevölkerung hervor und wirft die Frage nach der Sicherheit der deutschen Kernkraftwerke auf. Allerdings bieten sich in beiden Fällen den Regierungsparteien sehr unterschiedliche Alternativen an. In der Policydebatte zeigt sich, dass 2011 ganz andere Optionen auf dem Tisch liegen, als das 1986 der Fall war. Dies trägt auch zur Erklärung bei, warum die Debatte nach Fukushima in Deutschland ganz anders geführt wurde als in den europäischen Nachbarländern. So zeigen Brouard et al. (2013) etwa auf, dass die Pro-Kernenergiepolitik in Frankreich sich weniger durch die Unterstützung der öffentlichen Meinung erklären lässt als durch die Tatsache, dass dieses Thema von den wichtigsten politischen Parteien lange gemieden wurde. Inhaltlich legt das hier vorgestellte Beispiel also nahe, dass die Verfügbarkeit alternativer Lösungen den politischen Wettbewerb im Falle der Energiewende entscheidend beeinflusst hat.

Unser Beispiel zeigt aber vor allem, wie mithilfe des vorgestellten Datensatzes Entwicklungen innerhalb eines Politikfelds feingliedrig analysiert werden können. Durch die Möglichkeit schnell und direkt auf relevante Dokumente zu (Unter-)Themen zugreifen zu können, eröffnet die Datenbank für qualitative Forschungsdesigns interessante Chancen (s. Hacker 1997; Shpaizman 2019). Insbesondere kann unsere Datenbank Forschenden bei der Fallauswahl helfen und bietet Möglichkeiten, dann auch relativ rasch mit dem gewählten Fall vertraut zu werden. Die Zusammenstellungen aller Informationen über diverse politische Agenden hinweg bieten somit einen möglichen Einstieg in die detaillierte Prozessanalyse.

\section{Schlussbemerkungen}

Im vorliegenden Beitrag haben wir eine neue, umfassende Datenbank vorgestellt, die alle Etappen des Policyprozesses in Deutschland erfasst. Die Datenbank bietet 
ungemein vielseitige Möglichkeiten, Agendasetzungsforschung und -daten in politikwissenschaftliche Debatten einzubinden. Die Daten sind so aufbereitet, dass jede Beobachtung eindeutig identifiziert werden kann und damit auch mit weiteren Datenquellen, zum Beispiel zum politischen Wettbewerb, zusammengeführt werden kann.

Die kurze Beschreibung aller Daten bietet eine Vogelperspektive über das Aufkommen und Abklingen von politischen Themen in der Bundesrepublik und deren unterschiedliche Resonanz in verschiedenen politischen Agenden. Unsere Hoffnung ist es, dass dieser knappe Überblick die Reichhaltigkeit und den Umfang der Daten skizziert und darüber hinaus WissenschaftlerInnen und BürgerInnen anregt, die Datenbank für ihre eigenen (Forschungs-)Interessen zu nutzen. Unser Beispiel zur Energiepolitik umreißt das Potenzial der feingliedrigen Daten und der damit verbundenen Recherche für qualitative Prozessanalysen. Ungeachtet des einzelnen Forschungsdesigns freuen wir uns auf eine rege Nutzung dieses öffentlichen Guts.

Danksagung Wir danken allen wissenschaftlichen Hilfskräften, die an dem Projekt beteiligt waren, sowie Benjamin Guinaudeau und Miriam Hänni für Ihre Unterstützung und Mitarbeit. Christoffer GreenPedersen und Isabelle Guinaudeau stellten uns dankenswerterweise Parteidaten zur Verfügung. Schließlich danken wir den anonymen GutachterInnen sowie Kai-Uwe Schnapp für die konstruktive Kritik im Rahmen des Veröffentlichungsprozesses.

Förderung Das Projekt wurde durch das Canadian Social Sciences and Humanities Research Council, die Deutsche Forschungsgemeinschaft und die Universitäten Toronto und Konstanz gefördert.

Funding Open Access funding provided by Projekt DEAL.

Open Access Dieser Artikel wird unter der Creative Commons Namensnennung 4.0 International Lizenz veröffentlicht, welche die Nutzung, Vervielfältigung, Bearbeitung, Verbreitung und Wiedergabe in jeglichem Medium und Format erlaubt, sofern Sie den/die ursprünglichen Autor(en) und die Quelle ordnungsgemäß nennen, einen Link zur Creative Commons Lizenz beifügen und angeben, ob Änderungen vorgenommen wurden.

Die in diesem Artikel enthaltenen Bilder und sonstiges Drittmaterial unterliegen ebenfalls der genannten Creative Commons Lizenz, sofern sich aus der Abbildungslegende nichts anderes ergibt. Sofern das betreffende Material nicht unter der genannten Creative Commons Lizenz steht und die betreffende Handlung nicht nach gesetzlichen Vorschriften erlaubt ist, ist für die oben aufgeführten Weiterverwendungen des Materials die Einwilligung des jeweiligen Rechteinhabers einzuholen.

Weitere Details zur Lizenz entnehmen Sie bitte der Lizenzinformation auf http://creativecommons.org/ licenses/by/4.0/deed.de.

\section{Literatur}

Armingeon, Klaus (Hrsg.). 2013. Staatstätigkeiten, Parteien und Demokratie: Festschrift für Manfred G. Schmidt. Wiesbaden: Springer.

Bailer, Stefanie, Christian Breunig, Nathalie Giger, und Andreas Wuest. 2018. The diminishing value of minority representation: between group representation and individual career paths. Paper für die APSA-Konferenz, Boston.

Baumgartner, Frank, und Bryan Jones. 1993. Agendas and Instability in American Politics. Chicago: University of Chicago Press.

Baumgartner, Frank, und Bryan Jones. 2002. Policy dynamics. Chicago: University of Chicago Press.

Baumgartner, Frank R., Christian Breunig, Christoffer Green-Pedersen, Bryan D. Jones, Peter B. Mortensen, Michiel Nuytemans, und Stefaan Walgrave. 2009. Punctuated equilibrium in comparative perspective. American Journal of Political Science 53(3):603-620. 
Baumgartner, Frank R., Christian Breunig, und Emiliano Grossman (Hrsg.). 2019. Comparative agendas project: theory, tools, data. Oxford: Oxford University Press.

Behnke, Nathalie. 2009. Agenda-Setting für Verfassungsreformen. In Theorien der Verfassungsreform Jahrbuch für Handlungs- und Entscheidungstheorie, Bd. 5, Hrsg. Susumu Shikano, Joachim Behnke, und Thomas Bräuninger, 11-53. Wiesbaden: Springer VS.

von Beyme, Klaus. 1979. Die großen Regierungserklärungen der deutschen Bundeskanzler von Adenauer bis Schmidt. München: Carl Hanser.

von Beyme, Klaus. 1990. Politikfeldanalyse in der Bundesrepublik. In Politik in der Bundesrepublik Deutschland, Hrsg. Klaus von Beyme, Manfred Schmidt, 18-35. Opladen: Westdeutscher Verlag.

Bräuninger, Thomas, und Marc Debus. 2011. The policy agenda of political parties in multi-level systems: do German parties differ in their programmatic profile between the state and federal level? Paper vorgestellt bei der ECPR General Conference, Reykjavik, 25-27 August 2011.

Brettschneider, Frank. 2004. Agenda-Setting, Agenda-Cutting, Agenda-Surfing. Themenmanagement bei der Bundestagswahl 2002. In Der versäumte Wechsel. Eine Bilanz des Wahljahres 2002, Hrsg. Heinrich Oberreuter, 9-34. München: Olzog.

Breunig, Christian, Emiliano Grossman, und Tinette Schnatterer. 2019. Connecting government announcements and public policy. In Comparative agendas project: theory, tools, data, Hrsg. Frank Baumgartner, Christian Breunig, und Emiliano Grossmann. Oxford: Oxford University Press.

Brouard, Sylvain, Florent Gougou, Isabelle Guinaudeau, Simon Persico, und Alexandra Harwood. 2013. A campaign effect: the decline in French opposition to nuclear power in 2011-2012. Revue Française De Science Politique 63(6):19-49.

Budge, Ian, Hans Dieter Klingemann, Andrea Volkens, Judith Bara, und Eric Tanenbaum. 2001. Mapping policy preferences: estimates for parties, electors, and governments, 1945-1998. Oxford: Oxford University Press.

Cameron, David R. 1978. The expansion of the public economy: a comparative analysis. American Political Science Review 72(4):1243-1261.

Castles, Francis. 2002. Policy performance in the democratic state: an emergent field of study. In Comparative democratic politics, Hrsg. Hans Keman, 215-232. London: SAGE.

Cobb, Roger W., und Charles Elder. 1971. The politics of agenda-building: an alternative perspective for modern democratic theory. Journal of Politics 33:892-915.

Davies, Lincoln L. 2011. Beyond Fukushima: disasters, nuclear energy, and energy law. BYU Law Review. https://digitalcommons.law.byu.edu/lawreview/vol2011/iss6/5

Detlef, Jahn, und Sebastian Korolczuk. 2012. German exceptionalism: the end of nuclear energy in Germany! Environmental Politics 21(1):159-164.

Downs, Anthony. 1972. Up and down with Ecology-the Issue-Attention Cycle. The Public Interest 28:38-50.

Green-Pedersen, Christoffer, und Stefaan Walgrave. 2014. Agenda setting, policies, and political systems: a comparative approach. In Political agenda setting. An approach to studying political systems, Hrsg. Christoffer Green-Pedersen, Stefaan Walgrave, 1-16. Chicago: University of Chicago Press.

Greven, Michael. 2008. "Politik" als Problemlösung und als vernachlässigte Problemursache. Anmerkungen zur Policy-Forschung. In Die Zukunft der Policy-Forschung. Theorien, Methoden, Anwendungen, Hrsg. Frank Jannig, Kathrin Toens, 23-33. Wiesbaden: Verlag für Sozialwissenschaften.

Grimmer, Justin, und Brandon Stewart. 2013. Text as data: the promise and pitfalls of automatic content analysis methods for political texts. Political Analysis 21(3):267-297.

Guinaudeau, Isabelle. 2015. Documentation on the coding of German manifestos. Working paper, Sciences Po Bordeaux.

Hacker, Jacob. 1997. The road to nowhere: the genesis of president Clinton's plan for health security. Princeton: Princeton University Press.

Heichel, Stephan, Christoph Knill, Caroline Preidel, und Kerstin Nebel. 2015. Moralpolitik in Deutschland. In Moralpolitik in Deutschland. Analytische Besonderheiten und Implikationen für die weitere Forschung, Hrsg. Christoph Knill, Stephan Heichel, Caroline Preidel, und Kerstin Nebel, 243-263. Wiesbaden: Springer VS.

Jann, Werner, und Kai Wegrich. 2009. Phasenmodelle und Politikprozesse: Der Policy Cycle. In Lehrbuch der Politikfeldanalyse 2.0, 75-113. München: Oldenbourg.

Jannig, Frank, und Kathrin Toens (Hrsg.). 2008. Die Zukunft der Policy-Forschung. Theorien, Methoden, Anwendungen. Wiesbaden: VS.

John, Peter, Anthony M. Bertelli, Shaun Bevan, und William Jennings. 2013. Policy agendas in British politics. Comparative studies of political agendas. New York: Palgrave Macmillan. 
Jurka, Timothy P., Loren Collingwood, Amber E. Boydstun, Emiliano Grossman, und Wouter van Atteveldt. 2012. Rtexttools: automatic text classification via supervised learning. $R$ package version 1.3.9. http://CRAN.R-project.org/package=RTextTools. Zugegriffen: 6 Juni 2016

Klüver, Heike, und Jae-Jae Spoon. 2016. Who responds? Voters, parties, and issue attention. British Journal of Political Science 46(3):633-654.

Landwehr, Claudia, und Katharina Böhm. 2016. Strategic institutional design: two case studies of non-majoritarian agencies in health care priority-setting. Government and Opposition 51(4):632-660. https:// doi.org/10.1017/gov.2014.37.

Mortensen, Peter B., Christoffer Green-Pedersen, Gerard Breeman, Laura Chaqués-Bonafont, Will Jennings, Peter John, Anna M. Palau, und Arco Timmermans. 2011. Comparing government agendas executive speeches in the Netherlands, United Kingdom, and Denmark. Comparative Political Studies 44(8):973-1000.

Pampel, Fred C. 2011. Support for nuclear energy in the context of climate change evidence from the European Union. Organization \& Environment 24(3):249-268.

Proksch, Sven-Oliver, und Jonathan B. Slapin. 2015. The Politics of Parliamentary Debate. Cambridge: Cambridge University Press.

Rüb, Friedbert W. (Hrsg.). 2014. Rapide Politikwechsel in der Bundesrepublik. Theoretischer Rahmen und empirische Befunde. Baden-Baden: Nomos.

Rudzio, Wolfgang. 2005. Das politische System Deutschlands. Oldenburg: Springer VS.

Scharpf, Fritz. 1997. Games real actors play. Actor-centered institutionalism in policy research. Boulder: Westview Press.

Schmidt, Manfred G. 1987. West Germany: the policy of the middle way. Journal of Public Policy 7(02):135-177.

Schneider, Volker, und Frank Janning. 2006. Politikfeldanalyse. Akteure, Diskurse, und Netzwerke in der öffentlichen Politik. Wiesbaden: Springer VS.

Shpaizman, Ilana. 2019. Using CAP data for qualitative policy research. In Comparative agendas project: theory, tools, data, Hrsg. Frank Baumgartner, Christian Breunig, und Emiliano Grossmann. Oxford: Oxford University Press.

Stüwe, Klaus. 2002. Die großen Regierungserklärungen der deutschen Bundeskanzler von Adenauer bis Schröder. Opladen: Leske + Budrich.

Wenzelburger, Georg. 2015. Parties, institutions and the politics of law and order: how political institutions and partisan ideologies shape law-and-order spending in twenty western industrialized countries. British Journal of Political Science 45:663-687.

Wittneben, Bettina B. F. 2012. The impact of the Fukushima nuclear accident on European energy policy. Environmental Science \& Policy 15(1):1-3.

Wlezien, Christopher. 2017. Public Opinion and Policy Representation: On Conceptualization, Measurement, and Interpretation. Policy Studies Journal 45(4):561-582. 